\title{
Multi-mission operations with the Interferometry Science Center
}

\section{Andrew F. Boden, Rachel L. Akeson, Navid Dehghani, Jeff Jones, Kalyani Rengarajain, et al.}

Andrew F. Boden, Rachel L. Akeson, Navid Dehghani, Jeff Jones, Kalyani Rengarajain, Anneila I. Sargent, Quentin Sun, "Multi-mission operations with the Interferometry Science Center," Proc. SPIE 4844, Observatory Operations to Optimize Scientific Return III, (2 January 2002); doi: 10.1117/12.460753

Event: Astronomical Telescopes and Instrumentation, 2002, Waikoloa, Hawai'i, United States 


\title{
Multi-mission operations with the Interferometry Science Center
}

\author{
A.F. Boden ${ }^{a}$, R.L. Akeson ${ }^{a}$, N. Dehghani ${ }^{a}$, J. Jones ${ }^{a}$, K. Rengarajan ${ }^{a}$, A.I. Sargent ${ }^{a, b}$, Q. $\operatorname{Sun}^{a}$ \\ ${ }^{a}$ Interferometry Science Center, Caltech, 770 S. Wilson Ave, Pasadena, CA, USA \\ ${ }^{b}$ Astronomy Dept., Caltech, Pasadena, CA, USA
}

\begin{abstract}
The Interferometry Science Center (ISC) at the California Institute of Technology (Caltech) is chartered with providing science operations, data analysis support, and data archiving support for the suite of interferometry projects within the NASA Origins theme. Beginning with the Science Operations System (SOS) for the Keck Interferometer (KI), the ISC will design, implement, and operate a multi-mission facility to provide operations and support functions for NASA Origins interferometers and the scientists and engineers that use them. Future Origins interferometry projects such as the Space Interferometry Mission (SIM) will further use and extend the functionality of the ISC's multi-mission base. In this talk I will introduce the functional elements in the KI SOS; describe the common SOS core elements that KI and SIM will share; and provide prospective users of these facilities an introduction to user support model that the ISC is implementing.
\end{abstract}

Keywords: Interferometry, Science Data Processing System, Science Operations System

\section{INTRODUCTION}

NASA's Origins Theme seeks to address two defining science questions: where do we come from? - what are the origins of galaxies, stars, planets, and the chemical conditions necessary to sustain life; and are we alone? - exactly what are the physical and chemical properties necessary to sustain life, and how pervasive are those conditions on other planets*. As presently envisioned, a key technology in implementing the theme is longbaseline optical and infrared interferometry (herein OIR interferometry). OIR interferometry is a technology that combines light from two or more telescopes to obtain high-angular resolution information - see Shao \& Colavita $^{1}$ for a more extended discussion. Several of the key Origins missions will either definitely (e.g. Keck Interferometer, Space Interferometry Mission) or possibly (Terrestrial Planet Finder) use OIR interferometry as the instrument architecture to achieve high-angular resolution in pursuit of NASA Origins science objectives (see Colavita \& Wizinowich, ${ }^{2}$ Marr, ${ }^{3}$ and Hinz ${ }^{4}$ in these proceedings). Within the Origins theme the Navigator Program at the Jet Propulsion Laboratory manages the study and development of the OIR interferometry-based missions listed above, as well as other related activities.

Because the character and interpretation of OIR interferometer data can be complex and subtle, NASA has created a science operations and user support organization for the Navigator Program, the Interferometry Science Center (ISC), at the California Institute of Technology. The ISC supports Navigator Program interferometry projects in their development and operations, and provides full life cycle support for the scientists and engineers who use these Navigator Program facilities. In particular, the ISC provides users with observing facility access through proposal solicitation and selection; observation planning support; data product production, access, and long-term archiving; and data analysis support through contact science consultation. A key objective in the implementation strategy of the ISC is to emphasize common characteristics of the OIR interferometer instruments and data analysis techniques. The interested reader can find more information on the mission and scope of the ISC at the website ${ }^{\dagger}$ and in the contribution to these proceedings by Sargent. ${ }^{5}$

(Send correspondence to A.F.B: E-mail: bode@ipac.caltech.edu, Telephone: 626/395-1819)

${ }^{*}$ The interested reader is referred to http://origins.jpl.nasa.gov.

thttp://isc.caltech.edu 
In order to accomplish these operational objectives, the ISC is responsible for developing, hosting, and operating the Science Operations Systems (SOS) for the above Navigator Program projects. Further, the architectures for these SOS are being designed so as to identify, emphasize, and exploit common elements among them and the facilities they serve. Consequently, the ISC has structured its design activities around the common elements in an activity known as the ISC core. This ISC core is not an SOS and does not function per say, but is instead a tool suite that when integrated with facility-specific adaptations is used to synthesize an instance of an SOS for an ISC-supported facility. However, rather than trying to design this core a priori and then apply it, the core is being designed in conjunction with the prototyping of the KI SOS and the requirements analysis for the SIM SOS.

In what follows we will discuss the present development status of the prototype SOS for the Keck Interferometer, the requirements analysis for the Space Interferometry Mission, and the generalization to the ISC core, which will eventually be used to serve as the kernel of all ISC-supplied SOSs.

\section{KECK INTERFEROMETER SCIENCE OPERATIONS SYSTEM}

The Keck Interferometer (KI) is a ground-based component of the NASA Origins theme, and a facility instrument that serves the NASA, Caltech, University of California, and University of Hawaii science communities. $\mathrm{KI}$ is being built with NASA sponsorship by a partnership of the Jet Propulsion Laboratory (JPL), the William M. Keck Observatory (WMKO), and the ISC/Caltech. Operational goals of the Keck Interferometer are:

- High-sensitivity visibility amplitude measurements in the near infrared

- Differential phase detection of short period exoplanets

- Mid-infrared detection of exo-zodiacal emission from stars in the solar neighborhood

- Narrow-field differential astrometry to detect and characterize massive planets orbiting stars in the solar neighborhood

- Near-infrared synthesis imaging

The reader can find more information about KI at http://huey.jpl.nasa.gov/keck, and in the contribution by Colavita \& Wizinowich. ${ }^{2}$

As indicated above, the KI SOS design, implementation, and operation is the responsibility of the ISC. A schematic diagram of the KI SOS design is shown in Figure 1; it delineates the SOS top-level components and their architectural relationship. For ease of reference the major components are enumerated in the figure and in the following list:

- 0.0 Proposal Solicitation and Selection - activities associated with solicitation, peer-review evaluation, selection, and notification for investigations with KI

- 1.0 Science Planning User Interface - tools and services available to assist users in formulating and submitting observation plans

- 2.0 Science Planning Database - repository of observation plans and executed observations

- 3.0 Science Planning and Scheduling - formulation of long and short-range observation mode plans, and detailed scheduling of observation plans

- 4.0 WMKO Operations - real-time acquisition of raw (Level-0) data, quick-look processing, nightly wrapup processing (including the production of internally-calibrated Level-1 data), and data transmission from Hawaii to ISC

- 5.0 Data Archiving and Processing - data ingestion from Hawaii, and production, archiving, and access to raw and derived data products 


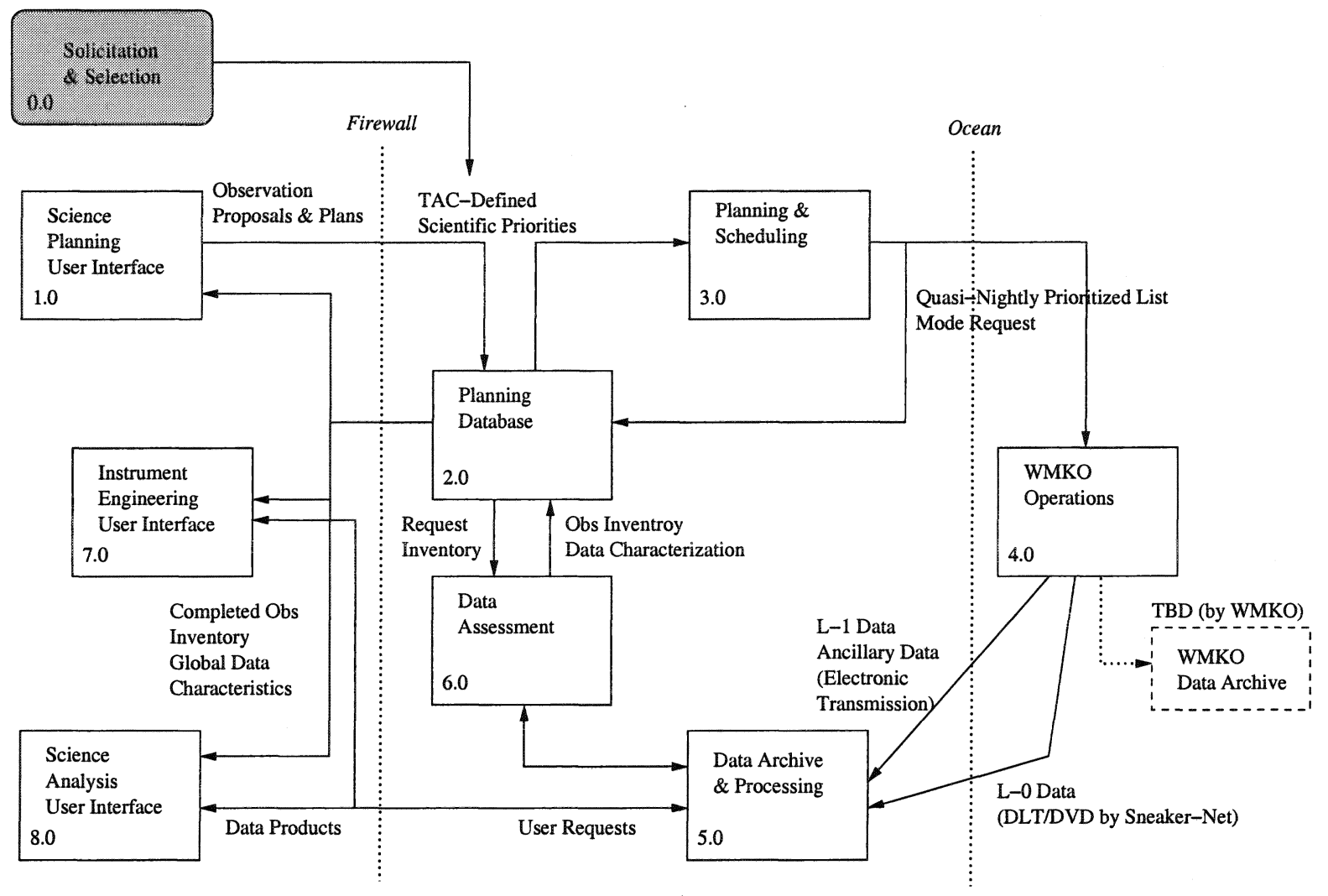

KI SOS -- Top Level

AB $1 / 02$

Figure 1. Schematic diagram of the Keck Interferometer Science Operations System (SOS).

- 6.0 Data Quality Assessment - determinations of data quality and monitoring of instrument performance

- 7.0 Instrument Engineering User Interface - tools and services to assist engineering users extraction and interpretation of instrument data products

- 8.0 Science Analysis User Interface - tools and services to assist science users extraction and interpretation of science data products

At this high-level of abstraction many of these functions appear very generic. Figure 2 gives an illustrative block diagram for the KI SOS Data Archiving and Processing component (5.0), in particular detailing the processing pipeline for the visibility amplitude $\left(V^{2}\right)$ and differential astrometry operating modes. Following these design activities the ISC is currently in development and prototype operations of the KI SOS in support of the present round of KI shared-risk science activities. ${ }^{2}$ Included in the current available user-support infrastructure is an observation planning package $\left(\mathrm{getCal}^{\dagger}\right)$, real-time reduction and visualizations, on-line data query, browsing, and access mechanisms (e.g. Fig. 3), $V^{2}$ data reduction (Kvis $\left.{ }^{\S}\right)$ and calibration (wbCalib

\footnotetext{
${ }^{\ddagger}$ http://isc.caltech.edu/software/getCal

${ }^{\S} \mathrm{http}: / /$ isc.caltech.edu/software/Kvis

『 http://isc.caltech.edu/software/wbCalib
} 


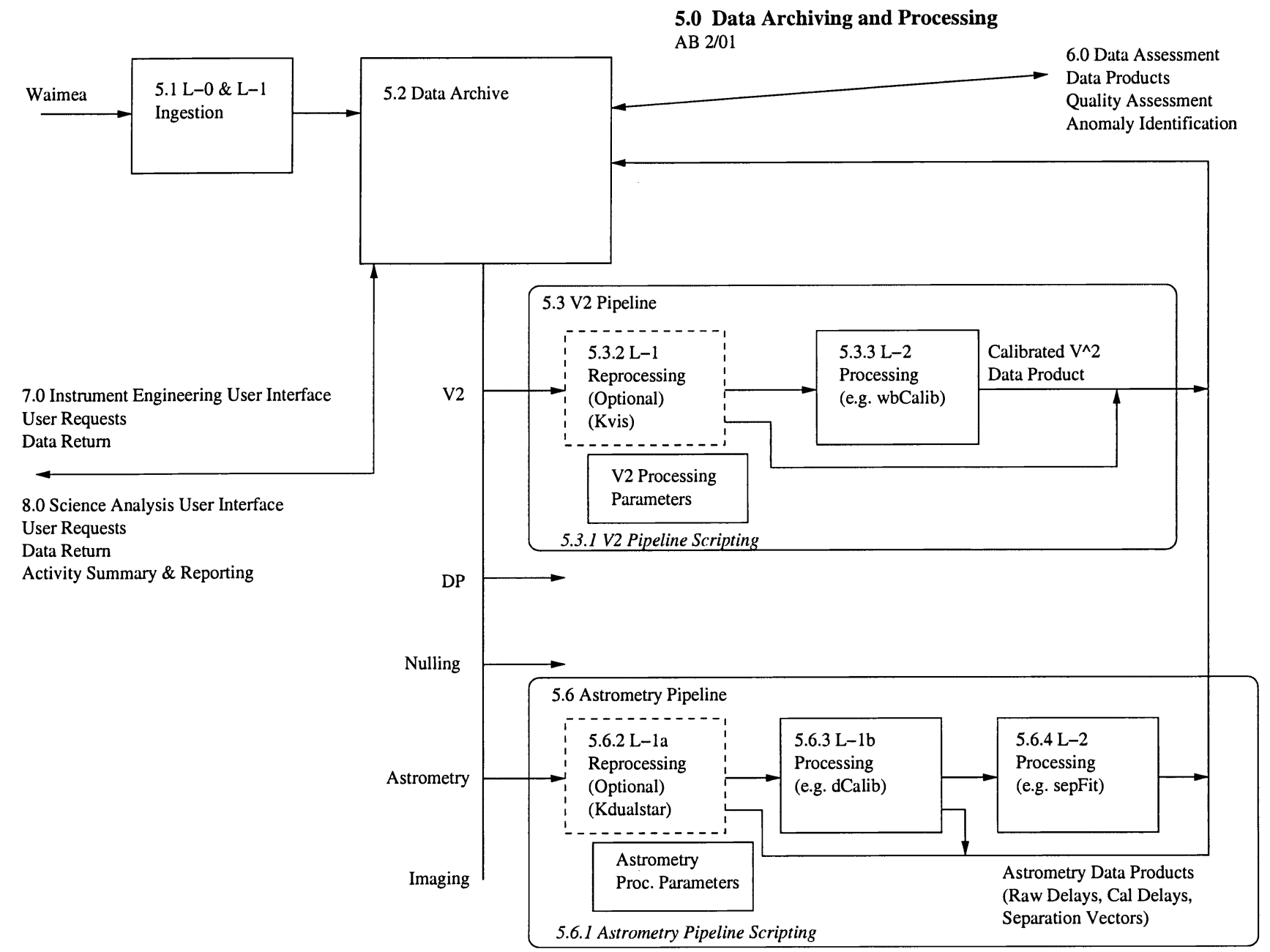

Figure 2. KI SOS Data Archiving and Processing Block Diagram 


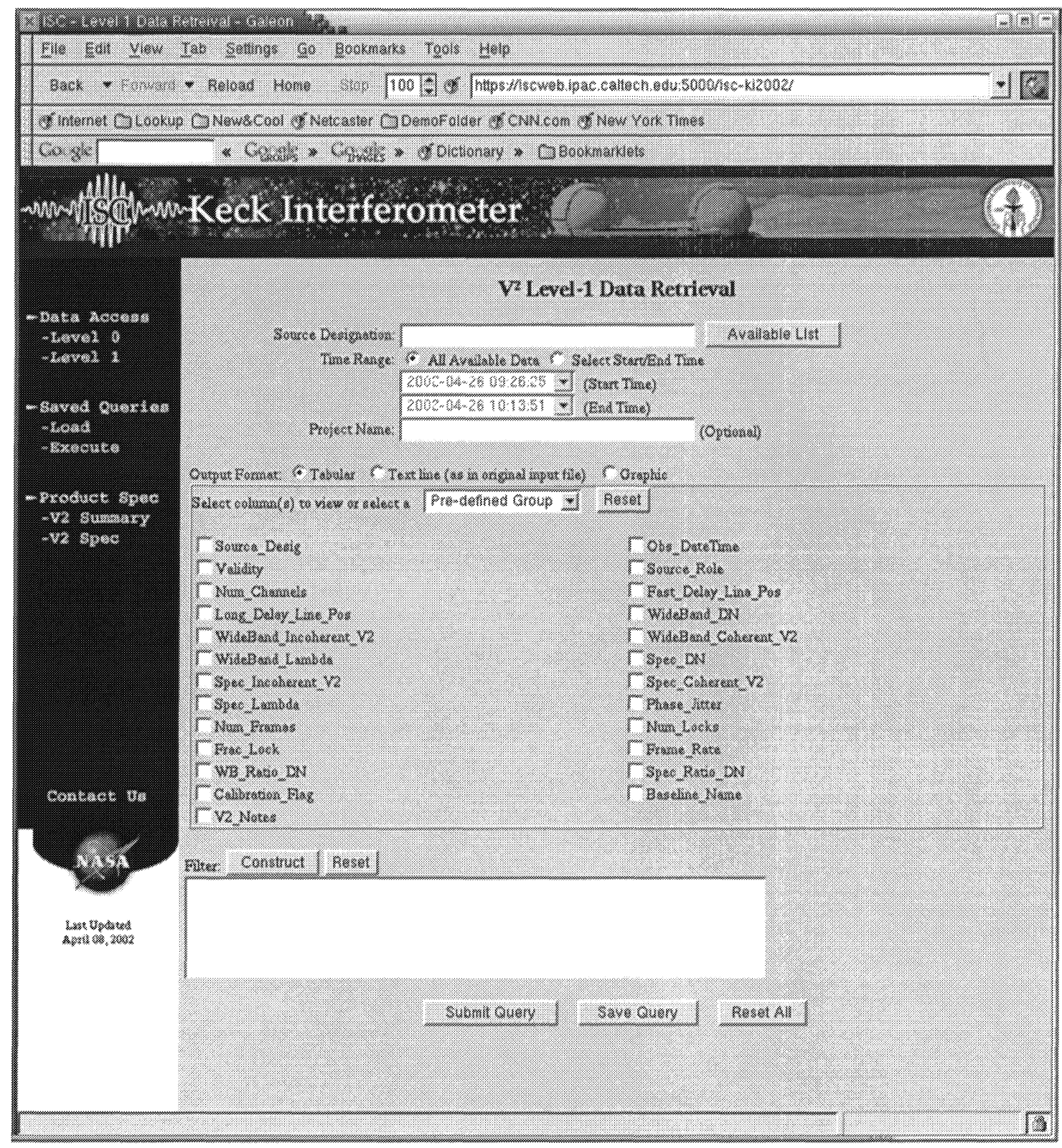

Figure 3. Screen Image of the ISC KI $V^{2}$ Level-1 Prototype Data Access Browsing and Extraction Interface

and $n b$ Cali $\left.^{\|}\right)$applications, and contact science support. Many of the support packages have extensive heritage from and use with the Palomar Testbed Interferometer (PTI, see Colavita $1999^{6}$ and Lane et al. $2002^{7}$ and references therein). The interested reader can find more information on KI support at the ISC web site http://isc.caltech.edu/KISupport.

\section{ABSTRACTION OF SOS COMPONENTS TO THE ISC CORE}

Proceeding concurrently with the development of the prototype KI SOS is the requirements and functional analysis of the SOS for the Space Interferometry Mission (SIM). Scheduled for launch in 2009, SIM will measure

\footnotetext{
"http://isc.caltech.edu/software/nbCalib
} 
the positions and motions of objects down to 20th magnitude at microarcsecond-class precision, and in particular will conduct a detailed survey of the frequency and architecture of planetary systems around pre-main sequence, main sequence, and evolved stars. The interested reader can find more information about SIM at http://sim.jpl.nasa.gov/ and the contributions by Marr ${ }^{3}$ and Shao. ${ }^{8}$

The SIM SOS will have similar top-level functions as the KI SOS. There will be similar proposal solicitation, submission, and selection mechanisms, science observation planning and science analysis interfaces. However, there will be notable differences with KI as well, for instance SIM scheduling constraints will be significantly different than KI, the downlink data from the spacecraft will be of higher granularity due to the limited communication bandwidth with the SIM spacecraft, and particularly the most important task in SIM's data analysis will the the construction of a full-sky astrometric reference model known as the SIM grid (see contributions by Shao ${ }^{8}$ and Swartz $^{9}$ ).

The fact that SIM design work is ongoing concurrently with KI prototype work offers a unique opportunity for the two designs to influence each other and helps to expose the common elements between the two systems. As mentioned above, the ISC is specifically chartered to implement these SOSs by emphasizing common elements realized as common implementations shared among the systems. Properly identified and codified, these core components offer obvious advantages both in terms of implementation and maintenance costs.

Based on our KI SOS prototype development and the ongoing analysis of the SIM SOS requirements, we have made an initial estimate of the common elements between KI and SIM, and allocated these elements to the ISC Core design. Figure 4 depicts this allocation in the context of the top-level KI SOS block diagram from Fig. 1. Generally components that are geared toward user interface (e.g. 0.0 Proposal Solicitation and Selection, 8.0 Science Analysis User Interface) are being designed to exploit the high degree of commonality among the OIR projects, while the lowest-level interfaces between the ISC and mission operations (e.g. 4.0 WMKO Operations) are highly mission-specific. The list below gives more specific comments about the core elements represented in each KI SOS component:

- 0.0 Proposal Solicitation and Selection - 100\% Core: Proposal solicitation and ingestion activities will be tightly integrated with the ISC's integrated web services using common proposal format and handling mechanisms; an integrated team will be responsible for the logistical support for peer-review evaluation, selection, and notification for investigations across the various SOSs operated by the ISC.

- 1.0 Science Planning User Interface - 75\% Core: Mission-specific observation planning tools and services will be derived from common tool sets for accessing astronomical catalogs, on-line databases, and computation and visualization of interferometer-related concepts such as delay-line range and u-v coverage. Common mechanisms for expressing the observation plans will be developed in the context of the ISC core, and available as either downloaded software or ISC web services. Mission-specific aspects of instrument geometry and performance will be integrated into the planning tool sets. Contact science support is available as solicited by outside investigators.

- 2.0 Science Planning Database - 80\% Core: A common infrastructure for storing, retrieving, and updating observation plans will be developed as part of the ISC core; integration of mission-specific quality assessment information as observing program is executed and data are received and processed.

- 3.0 Science Planning and Scheduling - 80\% Core: A common infrastructure for generating efficient longrange plans and detailed observing schedules will be developed as part of the ISC core. Mission-specific attributes and scheduling constraints will be incorporated as this component is adapted for each SOS.

- 4.0 WMKO Operations - 15\% Core: The mission operations interface to WMKO is largely mission-specific, consisting of custom low-level archiving mechanisms and processing to higher-level data products. Core elements include a web-based quick-look data visualization system, pipeline scripting, and data ingestion components.

- 5.0 Data Archiving and Processing - 50\% Core: Data archiving and retrieval mechanisms are developed in the context of the ISC Core; the definition of derived data products, the software to produce them, and design of the storage schema is a mission-specific activity. 


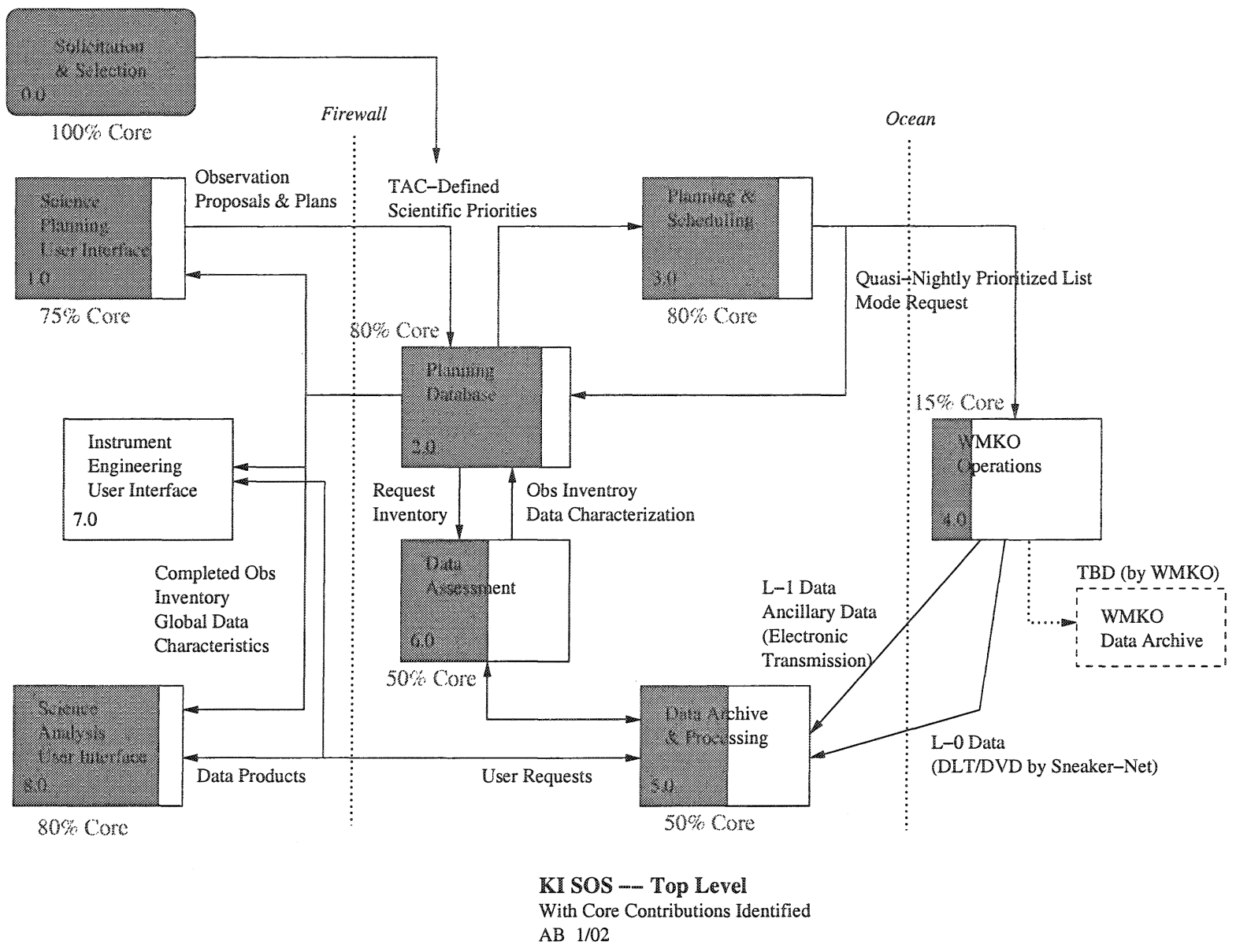

Figure 4. Schematic diagram of the Keck Interferometer SOS with ISC core contribution identified.

- 6.0 Data Quality Assessment - 50\% Core: The development of tools for data quality assessment and statistical characterization is a mission-specific responsibility, but the ISC core provides mechanisms for storing and retrieving these assessments and making them available to other components.

- 7.0 Instrument Engineering User Interface - 0\% Core: Purely a mission-specific activity based on our detailed interactions with the instrument development teams.

- 8.0 Science Analysis User Interface - 80\% Core: Visualization and retrieval of data products in a variety of standard formats (e.g. Flexible Image Transport System, FITS) is being developed largely in the context of the ISC core (e.g. Fig 3). Template analysis codes that illustrate the use of data products in limited science inference contexts will be available.

\section{SUMMARY}

The ISC is chartered with providing science operations, data analysis support, and data archiving support for the suite of interferometry projects within the NASA Origins theme. Beginning with the SOS for the Keck Interferometer (KI), the ISC is designing, implementing, and will operate a multi-mission facility to provide operations and support functions for NASA Origins interferometers and the scientists and engineers that use them. The users interface model the ISC is following is web-based; with electronic proposal solicitation and 
submission mechanisms, observation planning mechanisms, and data browsing and retrieval mechanisms. Enduser application codes that facilitate both observation planning and reduction will be available for download to run in the user's home environment. Finally, contact scientist support will be available to users through the entire life cycle of investigations with ISC-supported facilities.

\section{ACKNOWLEDGMENTS}

The work described in this paper is performed at the California Institute of Technology under the sponsorship of the National Aeronautics and Space Administration.

\section{REFERENCES}

1. M. Shao \& M. Colavita, "Long-baseline optical and infrared stellar interferometry" ARAछA 30, pp. 457498, 1992.

2. M. Colavita \& P. Wizinowich, "Keck Interferometer update", these proceedings 4838-10, 2002.

3. J. Marr, "Space Interferometry Mission (SIM) overview and current status", these proceedings 4852-01, 2002.

4. P.M. Hinz, N.J. Woolf, and J.R.P. Angel, "Spaced-based infrared interferometry: the quest for other solar systems", these proceedings 4852-22, 2002.

5. A.I. Sargent, "Interferometry Science Center (ISC)", these proceedings 4838-19, 2002.

6. M.M. Colavita et al., "The Palomar Testbed Interferometer", Ap.J 510, 505-521, 1999.

7. B.F. Lane, M.M. Colavita, and A.F. Boden, "PTI status report", these proceedings 4838-08, 2002.

8. M. Shao, "Science overview of the SIM project", these proceedings 4852-10, 2002.

9. R. Swartz, "SIM reference grid", these proceedings 4852-11, 2002 